\title{
Capacitance-Voltage Studies of Ti/p-ZnTe Schottky Barrier Structures Containing CdTe Quantum Dots
}

\author{
E. Placzek-Popko ${ }^{a}$, J. Szatkowski ${ }^{a}$, E. Zielony ${ }^{a}$, Z. Gumienny ${ }^{a}$, L. Dobaczewski $^{b}$ \\ AND G. KARCZEWSKI ${ }^{b}$ \\ ${ }^{a}$ Institute of Physics, Wrocław University of Technology, Wybrzeże Wyspiańskiego 27, 50-370 Wrocław, Poland \\ ${ }^{b}$ Institute of Physics, Polish Academy of Sciences, al. Lotników 32/46, 02-668 Warsaw, Poland
}

In this paper the electronic states of self-organized CdTe quantum dots embedded in ZnTe matrix are studied by means of capacitance-voltage $(C-V)$ characteristics within the temperature range of 180-300 K. A reference diode of the same layer structure but without quantum dots is studied also for comparison. The $C-V$ characteristics measured for the reference diode exhibit bulk behaviour in contrast to the quantum dots sample for which a characteristic step corresponding to discharging of quantum dots is clearly visible within broad range of temperatures. A quasistatic model based on the self-consistent solution of the Poisson equations is used to simulate the capacitance. By comparison the calculated $C-V$ curve with experimental curve the apparent thermal activation energy for hole emission from the quantum dots to the ZnTe matrix is found to be equal to $(0.12 \pm 0.03) \mathrm{eV}$.

PACS: 73.61.Ga, 73.21.La, 73.20.Hb

\section{Introduction}

Semiconductor quantum dots (QDs) have become very interesting for their unique optical and electronic properties. It is known that photoluminescence (PL) spectroscopy is a powerful tool for the determination of transition energies between electrons and holes in QDs. However it does not give information on their positions in the band gap. The $C-V$ spectroscopy can be used to investigate electron and hole levels positions separately in QDs by using $n$ - and $p$-type matrices. A comparison of experimental data with the calculated dependence of capacitance upon the applied voltage $(C-V)$ provides direct information about electronic structure of QDs [1].

$\mathrm{ZnTe}$ is a very promising candidate for green optoelectronics and integrated optics as it is the direct band gap semiconducting compound with a room temperature band gap equal to $2.26 \mathrm{eV}$ [2]. However it is well known that it is difficult to obtain $n$-type $\mathrm{ZnTe}$ and realize green light emitting diodes based on ZnTe. So far there was only a single report on such an achievement [3].

It has been accepted that $\mathrm{CdTe} / \mathrm{ZnTe}$ interface represents II type band alignment with CdTe valence band located above the $\mathrm{ZnTe}$ valence band, excluding the possibility of hole confinement for such an interface. However strain accompanying QD formation may lead to the inversion of alignment type, particularly in the case of the systems for which the band offset is close to zero, as it is in the case of the $\mathrm{CdTe} / \mathrm{ZnTe}$ system $[4,5]$. In present paper the capacitance spectroscopy has been used to study CdTe/ZnTe QDs system valence band offset and the evidence for hole confinement has been found.

\section{Samples and experimental details}

Two types of samples with the same layer structure were processed by molecular beam epitaxy method: a reference sample without quantum dots and a sample with self-assembled quantum dots. The QD sample consists of $3 \mu \mathrm{m}$ thick $p^{+}$-type ZnTe:N buffer deposited on the $p$-type GaAs substrate. On top of this structure a layer of CdTe QDs is sandwiched between $1 \mu \mathrm{m}$ layer of undoped ZnTe and $0.3 \mu \mathrm{m}$ of undoped ZnTe cap. The CdTe self-assembled QDs were grown in the Stranski-Krastanov mode by deposition of three monolayers of CdTe. The AuZnAu ohmic contacts were formed on the backside of GaAs substrate layer and the Schottky $\mathrm{Ti}(150 \mathrm{~nm})$ contacts were deposited by the photolithography method on the $\mathrm{ZnTe}$ cap layer.

$C-V$ measurements were performed within 180-300 K temperature range in a variable temperature cryostat with the help of Boonton capacitance meter operating at a frequency of $1 \mathrm{MHz}$.

\section{Results and discussion}

The $C-V$ characteristics measured for the reference diode exhibit bulk behaviour in contrast to the QDs sample for which a characteristic step corresponding to discharging of QDs is clearly visible within broad range of temperatures as it is shown in Fig. 1.

In the figure the $C-V$ characteristics for the $\mathrm{QD}$ diodes measured at different temperatures are shown. As temperature decreases the plateau associated with the QD discharge is suppressed. Such behaviour can be explained 


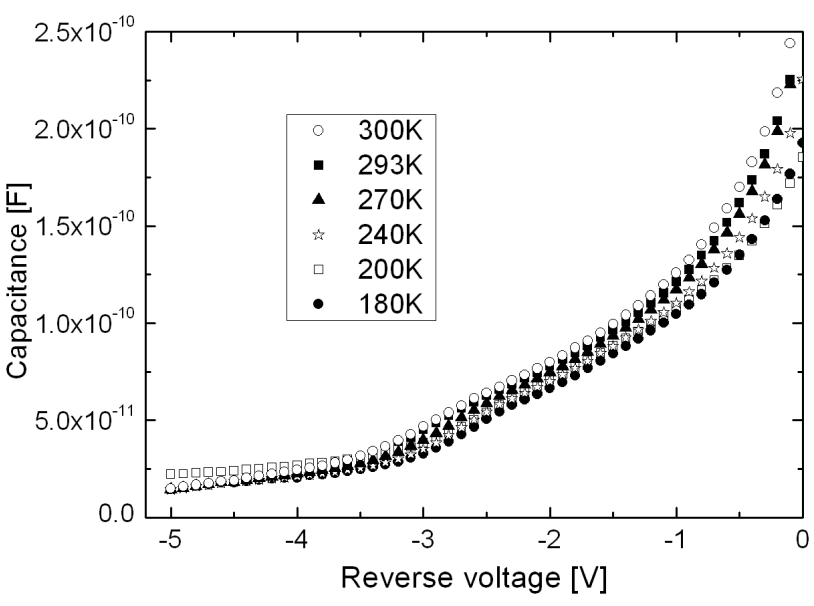

Fig. 1. $C-V$ curves for the $\mathrm{CdTe} / \mathrm{ZnTe}$ QD Schottky diode measured at different temperatures.

as follows. In the $C-V$ measurements ac signal of frequency $f$ is superimposed on the dc reverse bias. The ac signal modulates the charge at the edge of the space charge region (SCR) and at the point where the Fermi level crosses the hole level in QDs. The capacitance of the structure with quantum dots consists of two contributions

$$
C=C_{3 \mathrm{D}}+C_{\mathrm{qd}},
$$

the bulk capacitance $C_{3 \mathrm{D}}$ and capacitance $C_{\mathrm{qd}}$ associated with the $\mathrm{QD}$ layer. The capacitance $C_{\mathrm{qd}}$ is observed if the frequency measurement is low enough so that thermionic emission rate of holes from the QDs is much higher than $2 \pi f$. If the temperature decreases the emission rate decreases and the step in the $C-V$ characteristics becomes less significant.

A quasistatic model based on the self-consistent solution of the Poisson equations is used to calculate the $C-V$ curve [1]. The Poisson equation is solved for different values of reverse bias to obtain the charge in the structure per unit area. The calculated capacitance is derived from $C=\mathrm{d} Q / \mathrm{d} V$ based on quasistatic conditions, i.e., the temporal charge variation $\mathrm{d} Q$ caused by the increment of the reverse bias $\mathrm{d} V$ is neglected. The parameters used to fit the $C-V$ curves were obtained as follows. QD sheet density $N_{\mathrm{qd}}=10^{12} \mathrm{~cm}^{-2}$ was assumed to be equal to the density obtained from transmission electron spectroscopy (TEM) images of similar samples [6]. Spatial localization of the QDs was assumed to be equal to $0.3 \mu \mathrm{m}$, the ZnTe cap thickness. Shallow acceptor concentration $N_{\mathrm{A}}=4 \times 10^{16} \mathrm{~cm}^{-3}$ was determined from $C-V$ measurements. Built-in voltage could not be determined from the $C-V$ characteristics for high density of surface states. For this reason $V_{\mathrm{bi}}$ along with the QD binding energy were used as fit parameters. In order to obtain meaningful fit of the theoretical curve to the experimental data within whole measured reverse voltage bias a capacitance $C_{0}=85 \mathrm{pF}$ had to be extracted from the experimental data. It has to be pointed out, however, that there are defects in the studied diodes and due to their presence the apparent bulk capacitance measured at $1 \mathrm{MHz}$ frequency is different from the actual space charge region capacitance.

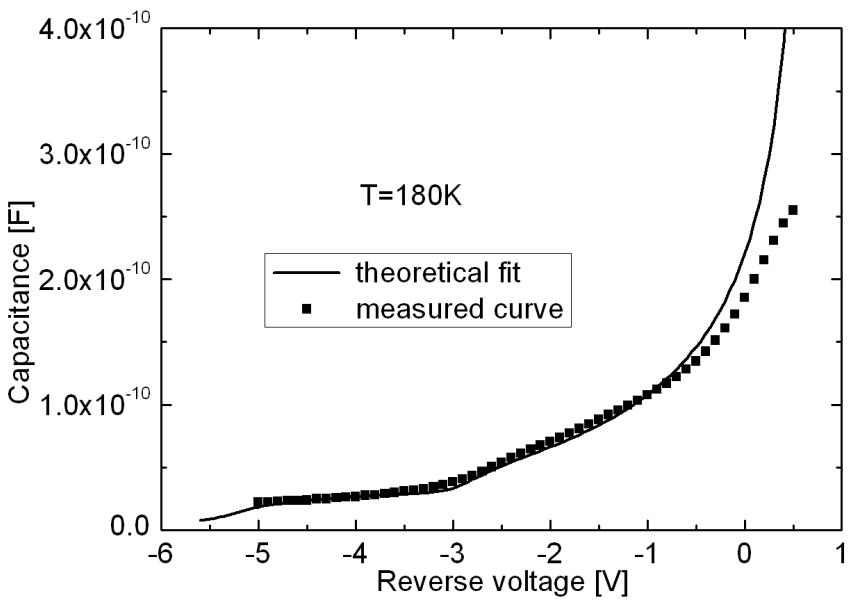

Fig. 2. Measured $C-V$ curve (squares) at $180 \mathrm{~K}$ and theoretical fit (solid line).

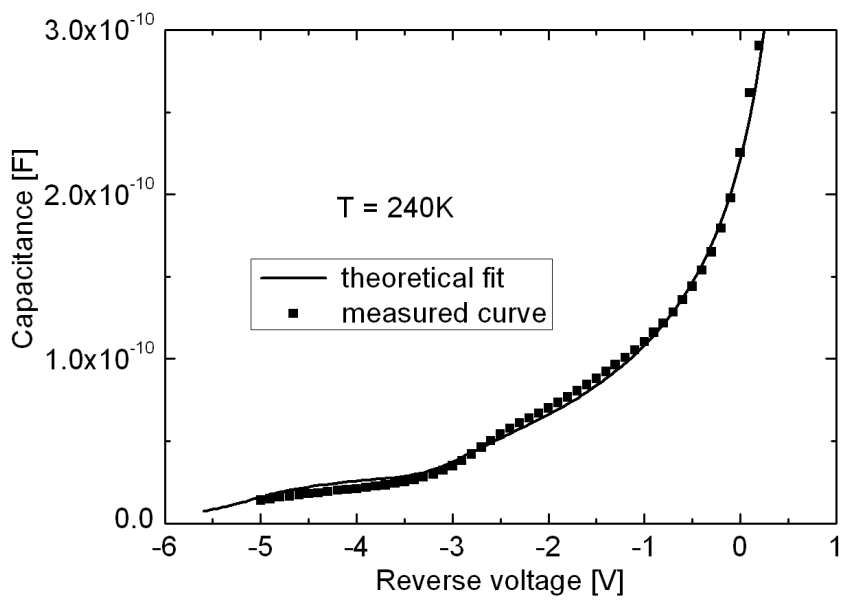

Fig. 3. Measured $C-V$ curve (squares) at $T=240 \mathrm{~K}$ and theoretical fit (solid line).

In Fig. 2 and in Fig. $3 C-V$ characteristics recorded at two different temperatures together with theoretical fits are displayed. In spite of the fact that the curves were measured at different temperatures (by $90 \mathrm{~K}$ ) both characteristics are well reproduced by theoretical fit with the same parameters: $V_{\mathrm{bi}}=0.7 \mathrm{~V}$ and the hole binding energy in QD $(0.12 \pm 0.3) \mathrm{eV}$.

\section{Conclusions}

In this paper the electronic states of self-organized CdTe QDs embedded in ZnTe matrix are studied by means of capacitance--voltage $(C-V)$ characteristics 
within the temperature range of 180-300 K. Two types of samples with the same layer structure were processed by molecular beam epitaxy method: a reference sample without quantum dots (Schottky $\mathrm{ZnTe}-\mathrm{Ti}$ ) and a sample with self-assembled CdTe quantum dots embedded in ZnTe matrix (Schottky $\mathrm{CdTe} / \mathrm{ZnTe}-\mathrm{Ti}$ ). The $C-V$ characteristics measured for the reference diode exhibit bulk behaviour in contrast to the QDs sample for which a characteristic step corresponding to discharging of QDs is observed within broad range of temperatures. A quasistatic model based on the self-consistent solution of the Poisson equations is used to calculate the capacitance [1]. By comparison with experimental data the energy level of the QDs is evaluated. It is found that the thermal activation energy for hole emission from the QDs to the ZnTe matrix equals $(0.12 \pm 0.03) \mathrm{eV}$. The quantum dot binding energy for holes of $0.12 \mathrm{eV}$ combined with the commonly observed energy of the excitonic luminescence from the $\mathrm{CdTe} / \mathrm{ZnTe}$ quantum dot system of around $2.0 \mathrm{eV}$ as measured at $T=10 \mathrm{~K}$ and compared to the $\mathrm{ZnTe}$ bandgap of $2.4 \mathrm{eV}$ (at $10 \mathrm{~K}$ ) allows to evaluate the quantum dot binding energy for electrons in the conduction band to be around $0.2 \mathrm{eV}$.

\section{Acknowledgments}

The work has been financially supported by the Ministry of Science and Higher Education project No. N202 091133.

\section{References}

[1] P.N. Brunkov, S.G. Konnikov, V.M. Ustinov, A.E. Zhukov, A.Yu. Egorov, V.M. Maximov, N.N. Ledentsov, P.S. Kop'ev, Semiconductors 30, 492 (1996).

[2] H. Bellakhder, A. Outzourhit, E.L. Ameziane, Thin Solid Films 382, 30 (2001).

[3] K. Sato, M. Hanafusa, A. Noda, A. Arakawa, M. Uchida, T. Asahi, O. Oda, J. Cryst. Growth 214/215, 1080 (2000).

[4] A. Continenza, S. Massidda, Phys. Rev. B 50, 11949 (1994).

[5] B. Späth, J. Fritsche, F. Säuberlich, A. Klein, W. Jaegermann, Thin Solid Films 480-481, 204 (2005).

[6] G. Karczewski, S. Maćkowski, M. Kutrowski, T. Wojtowicz, J. Kossut, Appl. Phys. Lett. 74, 3011 (1999). 